\title{
Pharmacist prescribing in critical care: an evaluation of the introduction of pharmacist prescribing in a single large UK teaching hospital
}

\author{
Verity J Cross, ${ }^{1}$ James T Parker, ${ }^{2}$ Marie-Christine Y L Law Min, ${ }^{2}$ Richard S Bourne ${ }^{2}$
}

${ }^{1}$ Department of Pharmacy, Sheffield Teaching Hospitals NHS Foundation Trust, Northern General Hospital, Sheffield, UK

${ }^{2}$ Departments of Pharmacy and Critical Care, Sheffield Teaching Hospitals NHS Foundation Trust, Northern General Hospital, Sheffield, UK

\section{Correspondence to}

James T Parker; james.parker@ sth.nhs.uk

Received 17 March 2017 Revised 14 July 2017 Accepted 18 July 2017 Published Online First 9 August 2017

\section{EAHP Statement 1:}

Introductory Statements and Governance.

EAHP Statement 4: Clinical Pharmacy Services. EAHP Statement 5: Patient Safety and Quality Assurance.
Check for updates

\begin{tabular}{l}
\hline To cite: Cross VJ, Parker JT, \\
Law Min M-CYL, et al. \\
Eur J Hosp Pharm \\
2018;25:e2-e6. \\
\hline
\end{tabular}

\section{ABSTRACT}

Objectives To evaluate the introduction of pharmacist independent prescribing activity across three general critical care units within a single large UK teaching hospital. To identify the prescribing demographics including total of all prescriptions, number prescribed by pharmacists, reason for pharmacist prescription, range of medications prescribed, pharmacist prescribing error rate and the extent of pharmacist second 'clinical check'. Methods Retrospective evaluation of e-prescribing across all general critical care units of a single large UK teaching hospital. All prescribing data were downloaded over a 1-month period (May to June 2016) with analysis of pharmacist prescribing activity including rate, indication, therapeutic class and error rate.

Results In total, 5374 medicines were prescribed in 193 patients during the evaluated period. Prescribing pharmacists were available on the units on $60.4 \%$ (58/96) of days, during their working hours and accounted for $576 / 5374(10.7 \%)$ of medicines prescribed in $65.2 \%(126 / 193)$ of patients. The majority (342/576) of pharmacist prescriptions were for new medicines. Infections, central nervous system, and nutrition/blood were the top three British National Formulary (BNF) therapeutic categories, accounting for $63.4 \%$ (349/576) of all pharmacist prescriptions. The critical care pharmacist prescribing error rate was $0.18 \%$ (1/550).

Conclusions Pharmacist independent prescribers demonstrated a high degree and wide-ranging scope of prescribing activity in general critical care patients. Pharmacists contributed a significant proportion of total prescribing, despite less than full service coverage. Prescribing activity was also safe with a very low error rate recorded.

\section{INTRODUCTION}

Clinical pharmacists make an essential contribution to the safe and effective use of medicines in critically ill patients. ${ }^{1}$ Direct patient care via pharmacist medication review is an important resource for reducing medication errors and optimising medication use. ${ }^{2}$ Recommendations for changes to medication therapy related to these medication reviews have very high acceptance rates by critical care medical teams. ${ }^{3-6}$ Pharmacist prescribing to action the outcomes of their own medication reviews would be anticipated to reduce the workload of medical colleagues and improve efficiency. Moreover, pharmacist instigation of medication therapy planned by the multidisciplinary team may also offer advantages with respect to getting treatment

\section{What this paper adds}

What is already known on this subject

- Medication reviews undertaken by pharmacists in critical care patients frequently result in recommendations for medicines optimisation, which have high acceptance rates by medical staff.

- Most UK critical care pharmacists indicated that they would be practising independent prescribers in the specialty by 2017.

- Few data concerning actual scope and level of prescribing practice in UK critical care units are currently available.

What this study adds

- This single centre study found that critical care pharmacists undertook significant prescribing activity across a wide range of therapeutic areas.

- Pharmacist prescribing errors were extremely uncommon.

right the first time in specific areas, for example, therapeutic drug monitoring or drug dosing in multiorgan failure.

Pharmacists have been able to practise as independent prescribers in the UK since 2006.' Baqir et al reported the scope and safety of pharmacist prescribing practice in general ward patients in a UK setting. ${ }^{8}$

In critical care, a 2014 survey of UK critical care pharmacists identified that by 2017 , most pharmacists would be practising as prescribers. ${ }^{4}$ The survey also provided some indication of the perceived benefit, scope and place for pharmacist prescribing in this specialty. ${ }^{4}$ However, to date, there are limited data on actual prescribing practice by critical care pharmacists. ${ }^{9}$

The aim of the present study was to describe the current scope and safety of prescribing activity undertaken by critical care pharmacists after the recent introduction of this service in a single large UK teaching hospital.

\section{METHODS}

The evaluation of prescribing activity was conducted over a 1-month period (19 May-19 June 2016; 32 days) across three critical care units (CCUs) within a single large UK teaching hospital. The study period was dictated by the timing of local project approval. All participating units were general CCUs (one 
intensive care unit (ICU), one high dependency unit (HDU) and one mixed ICU/HDU) comprising a total of 42 beds. All general CCUs used electronic prescribing (MetaVision; iMDsoft, Massachusetts, USA). The e-prescribing database had been customised and checked by pharmacists and included default doses for most medicines. Specialist neuro and cardiac CCUs were excluded as electronic prescribing was unavailable. A team of critical care pharmacists provided the clinical pharmacy service across all the general and specialist critical care units of the trust. The pharmacists were working at foundation, advanced or mastery level in critical care pharmacy practice. ${ }^{10}$ At the time of evaluation, 5 of the 10 pharmacists were practising prescribers. In the general CCUs, routine care was provided by three pharmacists per weekday and one pharmacist at weekends; the prescribing status of each pharmacist did not influence their inclusion in the rota.

All prescribing data were downloaded directly from the e-prescribing system into an Excel database (Microsoft Office, Washington, USA) for manipulation, for example, identification of pharmacist prescriptions. Electronic medical notes were then individually reviewed by VC (principal author and non-prescribing clinical pharmacist at foundation level in critical care pharmacy practice ${ }^{10}$ ) for further details on the indication of pharmacist prescribing and data related to second pharmacist 'clinical check' activity. The indications for pharmacist prescribing were recorded and categorised according to a 2014 survey of critical care pharmacist practice ${ }^{3}$ and a 2014 study of pharmacist prescribing in general hospital practice. ${ }^{8}$ The medicine prescriptions were categorised according to the primary indication for prescribing with determination from the medical note entry.

For the purposes of investigating error rate, an error was defined as 'any prescription which was incorrect at the time of prescribing and therefore needed subsequent amendment'. ${ }^{2}$ All critical care pharmacists were requested to inform the lead investigator $(\mathrm{VC})$ of any pharmacist prescribing errors identified and details. VC also retrospectively reviewed all pharmacist prescriptions and medical notes to identify any errors.

Nutritional supplements (eg, carbohydrate drinks) and maintenance fluids without additives were excluded from analysis.

Descriptive statistics were used to report prescribing data. The study was designated and registered as a service evaluation with the clinical effectiveness unit at the trust (CEU no 7372).

\section{RESULTS}

Over the 1-month evaluation period, a total of 5374 individual medicine prescriptions in 193 patients were completed by all prescribers within critical care. Pharmacists contributed 576 of these medicine prescriptions, of which critical care pharmacist independent prescribers (PIPs) prescribed 550 medicines and a specialist nutrition PIP prescribed a further 26 medicines. Pharmacists therefore provided $10.7 \%$ of the prescribing workload (576/5374).

Most critical care patients (126/193 (65.3\%)) had at least one medicine prescribed for them by a pharmacist.

A second 'clinical check' was undertaken by another critical care pharmacist retrospectively in approximately half of all medicine prescriptions $(286 / 550(52 \%))$. The timescale of this second 'clinical check' was not recorded, and the second clinical checks were noted as having been performed if another pharmacist approved the prescription at any time within the patient's critical care stay. These second 'clinical checks' identified no errors. During full review of all pharmacist prescriptions by the investigating pharmacist (VC), one error was identified, providing an error rate of $0.18 \%(1 / 550)$ during the period evaluated. The
Table 1 Pharmacist prescribed medicines by the British National Formulary (BNF) therapeutic class ${ }^{11}$

\begin{tabular}{lll}
\hline Chapter of the BNF (10) & $\begin{array}{l}\text { Percentage of } \\
\text { all medicines }\end{array}$ & $\begin{array}{l}\text { No of prescribed } \\
\text { medicines (N=576) }\end{array}$ \\
\hline 5. Infections & 23.0 & 132 \\
4. Central nervous system & 18.9 & 109 \\
9. Nutrition and blood & 18.7 & 108 \\
\hline 2. Cardiovascular system & 14.7 & 85 \\
1. Gastrointestinal system & 8.2 & 47 \\
6. Endocrine system & 6.4 & 37 \\
3. Respiratory system & 2.6 & 15 \\
15. Anaesthesia & 2.6 & 15 \\
11. Eye & 2.1 & 12 \\
\hline 12. Ear, nose and oropharynx & 1.6 & 9 \\
13. Skin & 0.5 & 3 \\
7. Obstetrics, gynaecology and urinary tract & & \\
disorders & 0.3 & 2 \\
14. Immunological products and vaccines & 0.2 & 1 \\
\hline Other & 0.2 & 1 \\
8. Malignant disease and & & \\
immunosuppression & 0 & 0 \\
\hline 10. Musculoskeletal and joint diseases & 0 & 0 \\
\hline
\end{tabular}

single error identified was classified as 'wrong dose'. The patient received an unadjusted paracetamol dose despite acute weight loss to $<50 \mathrm{~kg}$; this error was rectified by another pharmacist the following day.

The PIPs prescribed medicines from a wide range of therapeutic classes (table 1). The only two chapters of the British National Formulary $(\mathrm{BNF})^{11}$ not prescribed from were chapters 8 (malignant disease and immunosuppression) and 10 (musculoskeletal and joint disease).

The rationale for pharmacist prescribing was investigated (Figure 1), and the categories used to classify the data were derived from a questionnaire distributed to critical care pharmacist prescribers in $2014 .{ }^{4}$ Some of the categories were amended when the results began to show recurring themes, for example, antibiotics was split into 'new antibiotics' and 'antibiotic dose changes'.

The indications for prescribing were further categorised into four main groups, enabling direct comparison to the 2014 study of pharmacist prescribing in general hospital practice ${ }^{8}$ (table 2). This demonstrated that while the most frequent prescription indication on a general ward was 'regular medication not prescribed', the majority $(59.4 \%(342 / 576))$ of the critical care pharmacist prescribing activity was related to commencing new medicines.

\section{DISCUSSION}

To our knowledge, this is the first study that has specifically evaluated the practice of prescribing pharmacists in UK CCUs. Critical care PIPs contributed a significant proportion of total prescriptions, with a wide scope of practice, prescribing mostly new medicines with a very low error rate. This single centre evaluation benefits from a data analysis of pharmacist prescribing with more than 30 days of continuous data, 7 days per week across three general CCUs.

Approximately 11\% (576/5374) of all prescriptions during the evaluation period were prescribed by pharmacists, despite PIPs covering only approximately 60\% (58/96) of clinical pharmacy shifts across the CCUs during the study period, reflecting 
Reasons for prescribing as a percentage of total prescriptions

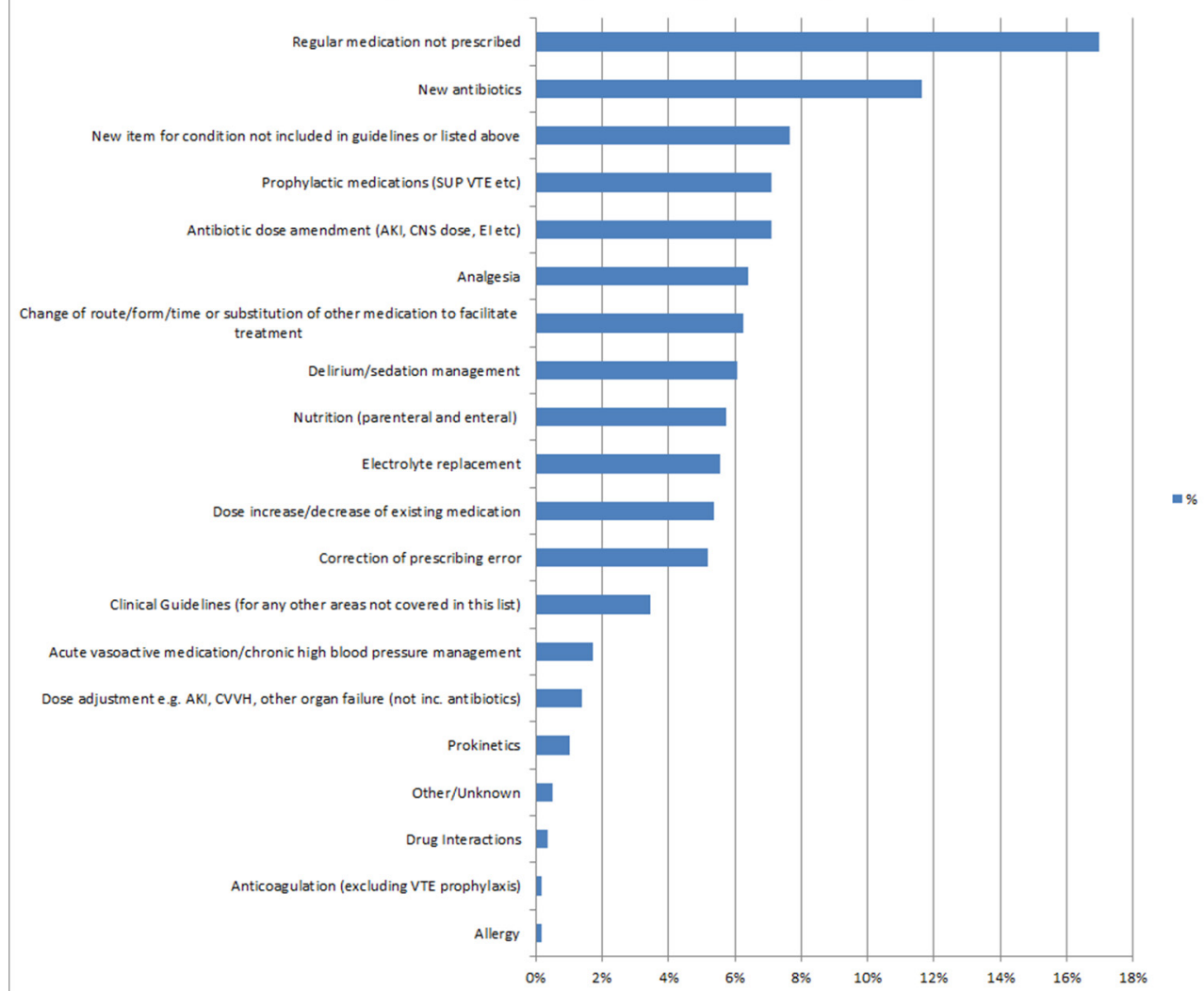

Figure 1 Graph demonstrating the indications for pharmacist prescribing expressed as a percentage of total pharmacist prescriptions. AKI, acute kidney injury; CNS, central nervous system; CVVH, continuous venovenous haemofiltration; El, extended infusion; SUP, stress ulcer prophylaxis; VTE, venous thromboembolism.

the incomplete introduction of this relatively new service. Pro rata this suggests that this figure could rise between $15 \%$ and $20 \%$ with a 'full' PIP service-where PIPs cover $100 \%$ of all clinical pharmacy shifts across the CCUs. This proportion is significantly higher than the results of a national 2014 survey of critical care pharmacist prescribing, ${ }^{4}$ where $90 \%$ of pharmacists estimated that their practice accounted for $10 \%$ or less of all new prescriptions.

Only 52\% (286/550) of PIP prescriptions received a second 'clinical check' by another pharmacist during the study. Throughout the evaluation, a single pharmacist was assigned to each CCU-often for up to 5 days to allow for service continuity. The availability of a second pharmacist on the unit to provide 'clinical checking' was therefore not routine. This,

Table 2 Comparison of critical care and general ward report by reason for medicine prescribing

\begin{tabular}{llc}
\hline \multirow{2}{*}{ Reason for prescribing } & \multicolumn{2}{c}{ No of medicines (\%) } \\
\cline { 2 - 3 } New medicine & Critical care & General ward (8) \\
\hline Medicine dose change & $59.4 \%(342)$ & $18.7 \%$ \\
Regular medication not prescribed & $18.6 \%(107)$ & $1.5 \%$ \\
Incorrectly prescribed medicines & $16.8 \%(97)$ & $68.1 \%$ \\
\hline
\end{tabular}

Critical care pharmacist independent prescribers (PIPs) covered these units on $60.4 \%(58 / 96)$ of days during normal working hours (08:00 to 16:30). These data suggest that each PIP prescribed an average of 10 medicines/day, although this varied greatly according to individual practitioner and availability of multiple pharmacists (0-44 medicines/day). combined with high patient turnover and the fact that delayed 'self-checking' was not included, may account for the relatively low rate of 'clinical checking' seen for PIP prescriptions. As such, pharmacist prescribers may be less likely to benefit from the safety support clinical pharmacists routinely provide to other prescribers.

The low error rate of pharmacist prescribing is comparable to other reports of pharmacist prescribing error rate ranging from $0 \%$ to $1.2 \% .^{812} 13$ The error in this study was identified retrospectively by the investigating pharmacist and not self-reported by the PIP. The error rate in the study may have been positively influenced by the e-prescribing system having default dosing for many drugs, and the fact that the principal investigator (VC) reviewing the prescriptions was a relatively junior critical care pharmacist. No PIP errors were identified by other pharmacists throughout the study, although pharmacists were instructed to report any errors seen throughout the data collection period.

This error rate compares favourably with medical staff prescribing, with large studies suggesting error rates between $7 \%$ and $9 \% .^{21415}$

The percentage of pharmacist prescriptions needed to correct a prescribing error was lower than the comparable study on general wards $(5.2 \%$ vs $7.5 \%) .{ }^{8}$ This may reflect the extent of the medication safety resources employed within critical care (e-prescribing, specialist critical care pharmacist 7 days/week, extensive critical care guidelines, education sessions, robust and responsive clinical incident reporting system ${ }^{16}$ and use of advanced nurse practitioners. ${ }^{17}$ 


\section{Medicines prescribed}

The General Pharmaceutical Council registrant survey in $2013^{18}$ reported that pharmacists most commonly prescribe antibiotics, analgesia, anticoagulation and cardiovascular medications. Antibiotics and cardiovascular medications have proven to be a recurring theme, ${ }^{19} 20$ as well as gastrointestinal, respiratory and eye preparations. A multicentre study in Northumbria ${ }^{8}$ revealed that items were prescribed by PIPs from all areas of the BNF except chapters 8, 14 and 15 (Malignant disease and immunosuppression, Immunological products and vaccines and Anaesthesia). In this critical care study, pharmacists prescribed from a wide range of therapeutic classes, most notably in the areas of infections, central nervous system, blood/nutrition and cardiovascular system disorders. There were no prescriptions for BNF chapters 8 and 10-malignancy/immunosuppression and musculoskeletal disorders. This may reflect critical care pharmacists working within their competencies or perhaps simply patient demographics over the evaluation period.

The reasons why pharmacists are prescribing were classified by two methods to facilitate useful comparison with existing reports of pharmacist prescribing in acute hospital patients. ${ }^{48}$

A survey of UK critical care pharmacist prescribing activity undertaken in $2014^{4}$ reported that the primary indications respondents prescribed for were: dose adjustment, change of medication route or formulation and correction of prescribing error (in decreasing order). ${ }^{4}$ Using comparable categories, the results of this evaluation indicated that the top three reasons for prescribing within the CCUs were 'regular medication not prescribed', 'new antibiotic' and 'new medicine for condition not listed in trust guidelines'.

Though there initially seems to be very little overlap between the questionnaire results and the data captured on the CCUs, this is in part owing to having two categories involving dose amendment ('antibiotic dose amendment' and 'dose adjustment not including antibiotics'), adding these categories together makes 'dose adjustment' a top three indication.

The high frequency of antibiotic prescriptions resulted in two antibiotic categories ('new antibiotic' and 'antibiotic dose amendment') to provide further information on pharmacist prescribing in practice. On all units, pharmacists attended the multiprofessional ward rounds including microbiology reviews. During these reviews, changes in choice of antimicrobial therapy are made in line with antimicrobial stewardship principles. Attending these daily ward rounds gives the pharmacists the most current information about the most appropriate antibiotics to use in specific patients, and they are then able to amend or change the antimicrobial prescription, utilising pharmacokinetic and pharmacodynamic principles, for example, employing effective loading doses as well as extended infusions of beta-lactam agents in order to optimise therapeutic plasma levels and facilitate their time-dependent bactericidal activity. ${ }^{21}$

Indeed, antimicrobials accounted for approximately $25 \%$ $(132 / 576)$ of all prescriptions, suggesting an important area of pharmacist prescribing practice and a clear role for all professionals in the treatment of infections: medical/nursing staff identify infection/sepsis; microbiologists guide therapy and clinical pharmacists optimise therapy.

Baqir et al reported a multicentre study of pharmacist prescribing in acute hospital inpatients receiving general ward care. ${ }^{8}$ A notable difference between the results of this critical care study and that undertaken in ward-based patients ${ }^{4}$ is that critically ill patients receive a higher rate of new medicines from pharmacists $(59.4 \%$ vs $18.7 \%)$ rather than continuity of regular medicines not prescribed (16.8\% vs $68.1 \%)$. The acuity of critical care patient therapeutics means more frequent assessments, and changes in therapy are required and these include medication reviews and prescribing outcomes. This may partly explain why pharmacists working in critical care prescribed medicines for approximately two-thirds of patients rather than the less than forty per cent reported by Baqir et $a l .{ }^{4}$ In this study of critical care patients, dose adjustment explained approximately one-fifth $(107 / 576)$ of pharmacist prescriptions but only a tiny minority $(1.5 \%)$ of those reported in ward-based patients. ${ }^{4}$ The severity of illness of critical care patients and frequency of multiorgan failure explains this difference compared with general ward patient care. The percentages of prescriptions written owing to 'incorrectly prescribed medications' were comparable (5.2\% and $7.5 \%$, respectively), although the percentage on general wards was slightly higher, as already discussed.

The data presented in this evaluation are arguably an underestimate of pharmacist prescribing within the CCUs for several reasons. The utilisation of the e-prescribing system had some notable limitations inherent to the system used. First, deprescribing is not searchable within the main database system. Discontinuation of medicines no longer indicated or that are inappropriate is an important prescribing activity that we are unable to report in the study. Second, medicines prescribed but not administered are similarly unrecordable (eg, 'when required' medications never administered do not register as having ever been prescribed).

In addition to the above, within this evaluation, no differentiation was made between transcribed medications (medications which had been started prior to critical care admission) and new prescriptions (medications initiated on critical care for an acute illness); the critical care units use e-prescribing, but the rest of the hospital use handwritten paper charts. All prescriptions on the e-prescribing system appear to be 'new' regardless of the actual start date, and it was not possible to retrospectively differentiate between transcribed and newly initiated medications. Pharmacists do not generally transcribe medications (transfer ward drug cards onto the electronic system) as this is considered the role of the junior doctors or advanced nurse practitioners; therefore, if transcribed medications were excluded from analysis, the proportion of prescriptions written by PIPs would likely increase. Overall, these limitations mean that the extent of critical care pharmacist prescribing as a percentage of all prescriptions is likely to be an underestimate.

\section{CONCLUSION}

Critical care pharmacists contributed a significant proportion of total prescriptions, with a wide scope of practice, prescribing mostly new medicines with a very low error rate.

Further multicentre studies are required to identify areas where critical care pharmacists should lead prescribing and medicines optimisation and how this impacts on patient outcomes.

Contributors All four authors (VC, JTP, M-CYLLM and RSB) contributed to the design of the work, data collection, analysis and interpretation; drafted the manuscript and revised it critically for important intellectual content; provided final approval of the version published and agreed to be accountable for all aspects of the work in ensuring that questions related to the accuracy or integrity of any part of the work are appropriately investigated and resolved. There are no other contributors to be acknowledged in the publication of this manuscript.

Competing interests None declared.

Provenance and peer review Not commissioned; externally peer reviewed. 
(C) European Association of Hospital Pharmacists (unless otherwise stated in the text of the article) 2018. All rights reserved. No commercial use is permitted unless otherwise expressly granted.

\section{REFERENCES}

1 Chant C, Dewhurst NF, Friedrich JO. Do we need a pharmacist in the ICU? Intensive Care Med 2015;41:1314-20.

2 Shulman R, McKenzie CA, Landa J, et al. Pharmacist's review and outcomes: treatment-enhancing contributions tallied, evaluated, and documented (PROTECTEDUK). J Crit Care 2015;30:808-13.

3 Klopotowska JE, Kuiper R, van Kan HJ, et al. On-ward participation of a hospital pharmacist in a Dutch intensive care unit reduces prescribing errors and related patient harm: an intervention study. Crit Care 2010;14:R174.

4 Bourne RS, Whiting P, Brown LS, et al. Pharmacist independent prescribing in critical care: results of a national questionnaire to establish the 2014 UK position. Int J Pharm Pract 2016;24:104-13.

5 Bourne RS, Choo CL, Dorward BJ. Proactive clinical pharmacist interventions in critical care: effect of unit speciality and other factors. Int J Pharm Pract 2014;22:146-54.

6 Richter A, Bates I, Thacker M, et al. Impact of the introduction of a specialist critical care pharmacist on the level of pharmaceutical care provided to the critical care unit. Int J Pharm Pract 2016;24:253-61.

7 Baqir W, Miller D, Richardson G. A brief history of pharmacist prescribing in the UK. Eur J Hosp Pharm 2012;19:487-8.

8 Baqir W, Crehan O, Murray R, et al. Pharmacist prescribing within a UK NHS Hospital Trust: nature and extent of prescribing, and prevalence of errors. Eur I Hosp Pharm 2015;22:79-82.

9 Bourne RS, Baqir W, Onatade R. Pharmacist independent prescribing in secondary care: opportunities and challenges. Int I Clin Pharm 2016:38:1-6.

10 Department of Health Adult Critical Care - Specialist Pharmacy Practice London, 2005:1-27 www.codeg.org/fileadmin/codeg/pdf/Critical_care_specialist_ guidance.pdf (accessed 14 Jun 2017).
11 Joint Formulary Committee. British National Formulary. London: BMJ Group and Pharmaceutical Press, 2016:71.

12 Harrogate, UK.Cerrato M, Pearce S. Pharmacy department, University Hospitals Southampton NHS Foundation Trust, Southampton. impact of pharmacist prescriber service to the cardiovascular and thoracic (CV\&T) care group. Poster presentation at the GHP/ UKCPA Joint National Conference 2013.

13 Nicholls JS, Butt M, Ogden D, et al. Management of patients with HIV-1 infection by pharmacist prescribers: an evaluation of practice. Eur J Hosp Pharm 2014:21:13-17.

14 Ryan C, Ross S, Davey P, et al. Prevalence and causes of prescribing errors: the PRescribing Outcomes for Trainee doctors Engaged in Clinical Training (PROTECT) study. PLoS One 2014;9:e79802.

15 Dornan T, Ashcroft D, Heathfield H, et al. An in depth investigation into causes of prescribing errors by foundation trainees in relation to their medical education EQUIP study. London: General Medical Counsel, 2009. http://www.gmc-uk.org/FINAL_ Report_pr.

16 Bourne RS, Shulman R, Jennings JK. Important resources to reduce medication errors in critical care patients and classification of units: a UK clinical pharmacist consensus. Int J Pharm Pract 2017.

17 Carberry M, Connelly S, Murphy J. A prospective audit of a nurse independent prescribing within critical care. Nurs Crit Care 2013;18:135-41.

18 Phelps A, Agur M, Nass L, et al. GPhC Registrant survey 2013. General Pharmaceutical Council 2014.

19 Hinchliffe A. Pharmacist independent prescribing - a review of the evidence. 12: Public Health Wales NHS Trust, 2015.

20 Manchester, UK.Adaeddine S, Khachi M, Milller G. Impact of pharmacist independent prescribers at Imperial College Healthcare NHS Trust. Poster presentation at GHP/ UKCPA 10th Joint National Conference 2014.

21 Cotta MO, Roberts JA, Lipman J. We need to optimize piperacillin-tazobactam dosing in critically ill patients-but how? Crit Care 2016;20:163. 